\title{
A dynamic discrete-choice model for movement flows
}

Link to publication record in Manchester Research Explorer

\section{Citation for published version (APA):}

Koskinen, J., Muller, T., \& Grund, T. (2017). A dynamic discrete-choice model for movement flows. In C. Perna, M. Pratesi, \& A. Ruiz-Gazen (Eds.), Studies in Theoretical and Applied Statistics (Vol. 227, pp. 107-117). Springer Nature.

\section{Published in:}

Studies in Theoretical and Applied Statistics

\section{Citing this paper}

Please note that where the full-text provided on Manchester Research Explorer is the Author Accepted Manuscript or Proof version this may differ from the final Published version. If citing, it is advised that you check and use the publisher's definitive version.

\section{General rights}

Copyright and moral rights for the publications made accessible in the Research Explorer are retained by the authors and/or other copyright owners and it is a condition of accessing publications that users recognise and abide by the legal requirements associated with these rights.

\section{Takedown policy}

If you believe that this document breaches copyright please refer to the University of Manchester's Takedown Procedures [http://man.ac.uk/04Y6Bo] or contact uml.scholarlycommunications@manchester.ac.uk providing relevant details, so we can investigate your claim.

\section{OPEN ACCESS}




\title{
A dynamic discrete-choice model for movement flows
}

\author{
Johan Koskinen, Tim Müller, and Thomas Grund
}

\begin{abstract}
We consider data where we have individuals affiliated with at most one organisational unit and where the interest is in modelling changes to these affiliations over time. This could be the case of people working for organisations or people living in neighbourhoods. We draw on dynamic models for social networks to propose an actor-oriented model for how these affiliations change over time. These models specifically take into account constraints of the system and allow for the system to be observed at discrete time-points. Constraints stem from the fact that for example not everybody can have the same job or live in the same neighbourhood, something which induces dependencies among the decisions marginally. The model encompasses two modelling components: a model for determining the termination of an affiliation; and a discrete-choice model for determining the new affiliation. For estimation we employ a Bayesian data-augmentation algorithm, that augments the observed states with unobserved sequences of transitions. We apply the proposed methods to a dataset of house-moves in Stockholm and illustrate how we may infer the mechanisms that sustain and perpetuate segregation on the housing market.
\end{abstract}

Key words: Social Networks, Stochastic actor-oriented models, Allocation models, Matching models

Johan Koskinen

Social Statistics Discipline Area, University of Manchester, Manchester M13 9PL, \& , Institute of Analytical Sociology, University of Linköping, Norra Grytsgatan 10, 60174 Norrköping, Sweden e-mail: johan.koskinen@manchester.ac.uk

Tim Müller

Berlin Institute for Integration and Migration Research, Humboldt-Universität zu Berlin, Unter den Linden 6,10099 Berlin e-mail: t.mueller@ sowi.hu-berlin.de

Thomas Grund, School Of Sociology, Newman Building, Belfield, Dublin 4, Ireland e-mail: thomas.grund@ucd.ie 


\section{Introduction}

In the economic, social, and behavioural sciences there is a long tradition of analysing why people end up in certain professions, particular schools, and different neighbourhoods. Standard statistical approaches such as discrete-choice models allow for investigating how different characteristics increase a person's likelihood to end up in one destination rather than another or how characteristics of the final destinations explain who ends up there. It has however been recognised that there are systemic properties of these phenomena that constrain and structure choices and destinations. For example, whereas everybody might want to go to the best school, one school cannot accommodate everyone (Abdulkadiroglu and Sonmez, 2003). When it comes to, say, occupations, typically (excluding individuals with for example multiple part-time jobs) people only have one occupation at any one time and the likelihood that an individual ends up in one job might be a function of their own preference as well as the composition of employees already in that job (Butts, 2007).

To accommodate the systemic dependencies and restrictions associated with an allocation system we formulate a constrained version of the stochastic actororiented modelling (SAOM) family for two-mode networks proposed by Koskinen and Edling (2012). The SAOM, originally developed for modelling the evolution of one-mode networks by Snijders (2001), models the dynamic network as a discrete Markov chain in continuous time, where changes to the network can be seen as incremental updates where the network changes one tie at a time. The process thus defined is a random walk on a $d$-cube (Aldous, 1983) for $d$ number of tievariables. A particularly attractive aspect of Snijders' (2001) modelling framework is that each incremental change to the network can be modelled as an independent discrete-choice model, conditional on the current state of the network (Snijders, 2006, additionally provided a model formulation that enjoys similar properties but that relaxes the assumption of choice). Furthermore, even if we only observe the network at discrete points in time, the SAOM provides us with a model for imputing or augmenting our observed data with any changes that might have occurred in-between observations. The extension to two-mode network data or, indeed, any system that affords dissagregating complex dependencies into a series of cumulative changes modelled as discrete-choice models is mostly a choice of appropriate restrictions and determining the decision process.

In the following we proceed by defining the data-structure that motivates our model. We then define the model in detail and outline the inference scheme. Finally we provide an illustrative example of applying the model to a data set that is a subset of a rich and very complex register data set for inhabitants in Sweden. 


\section{Data Structure}

We will distinguish between two types of social entities, individuals and their locations. Individuals may be alternatively people, organisations, or some other social entity that we are willing to endow with some form of decision-power. The locations will represent collections of individuals, such as organisations, sectors, etc. For the sake of our empirical setting we will refer to these two entities as individuals and neighbourhoods. Let $\mathscr{I}=\{1, \ldots, n\}$ be a set of individuals and $\mathscr{J}=\{1, \ldots, m\}$ a set of neighbourhoods. A binary variable $Y_{i j}(t)$ indicates whether $i \in \mathscr{I}$ lives in neighbourhood $j \in \mathscr{J}$ at time $t \in \mathbb{R}^{+}$.

We denote by $v_{i}(t)$ a $k \times 1$ vector of covariates of $i$ and $w_{j}(t)$ a vector of covariates for $j$. In addition we may have dyadic covariates for the individuals, e.g. $a_{i u}$ being one or zero according to whether there is a kinship tie between $i$ and $u$.

We assume that the matrix $Y=\left(Y_{i j}\right)_{(i, j) \in \mathscr{I} \times \mathscr{J}}$ is row-regular such that $Y_{i+}=$ $\sum_{j} Y_{i j}=1$ for all individuals. This eliminates homeless individuals and individuals registering multiple living addresses, as well as individuals moving to areas that are not in $\mathscr{J}$. For the purposes of defining the model, we need not necessarily distinguish between individuals being people or households. However, for the purpose of analysis, individuals may conveniently be collapsed into households. This prevents direct analysis of transition into cohabitation, children transitioning from dependants to independants, etc.

Data could be time-stamped in the sense that we observe the exact time at which every change occurs. In most cases we would however expect that there is some form of discretisation of observation times, for example that only the month or year of a change is known. For this reason we make the tacit assumption that data are observed at discrete points in time $t_{0}, t_{1}, \ldots, t_{M+1}$ but in order to account for the fact that changes do not happen simultaneously, we assume that there are unobserved time-points $s_{1, u}, \ldots, s_{K_{u}, u}$ in-between $t_{u-1}$ and $t_{u}$ at which changes are made to $Y$. For the purposes of inference we will assume that $Y_{i}$. may change at most once inbetween $t_{u-1}$ and $t_{u}$ for any $u=1, \ldots, M+1$. We use the notational conventions of letting $j\left(Y_{i \bullet}(t)\right)=\left\{j \in \mathscr{J}: Y_{i j}(t)=1\right\}$ and $t_{i}=t_{i}\left(\{Y(t)\}_{t \in[s, T]}\right)=\inf \{t \in[s, T]:$ $\left.Y_{i \bullet}(t) \neq Y_{i \bullet}(s)\right\}$.

We may construe the process of changes as a process of evolution of a bipartite network (Koskinen and Edling, 2012) subject to the constrains of row-regularity and parsimonious paths.

\section{Model Formulation}

We follow the schema of Snijders $(2001,2006)$ in defining the process in terms of waiting times and conditionally on this a discrete-choice model, When an individual $i$ chooses to move at, say, time $t$, we assume that $i$ has complete information about the entire state of the system and that $i$ may choose to move to any of the neighbourhoods in $\mathscr{J}$. We assume that $i$ associates with $j \in \mathscr{J}$ a utility 
$U_{i}(j ; Y(t), t, \theta)=f_{i}(j ; Y(t), t, \theta)+\varepsilon(i, j, t)$, where $f_{i}$ is an objective function that we aim to model and $\varepsilon(i, j, t)$ are random components that we will assume are i.i.d. extreme value type one distributed for all $i, j$, and $t$. We assume that the individuals maximise this function which implies (Maddala, 1983) that the conditional probability when a change is made conditional on the current state (at $t=t_{i}-\delta$ ) is given by

$$
\operatorname{Pr}\left(Y_{i j}\left(t_{i}\right)=1 \mid Y_{i \cdot(t)}\right)=\frac{e^{f_{i}(j ; Y(t), t, \theta)}}{\sum_{k \in \Omega(\mathscr{J} ; Y(t))} e^{f_{i}(k ; Y(t), t, \theta)}}
$$

where $\Omega(\mathscr{J} ; Y(t))$ denotes the option set. We shall limit the formulation of the systematic part of the utility function to being a weighted sum of statistics. The latter shall be functions of $Y, v, w$, and $a$.

While the conditional discrete choice model is the main focus, for purposes of specifying a generative model we also need to specify a process for picking who is moving. It the most general form, we assume that the rate at which an individual $i$ moves out of their current neighbourhood is $\lambda_{i}(Y(t), t, \theta)$, and that the holding-times are conditionally independent conditional on the current state $Y(t)$ and observable covariates. We may construct indicators $A_{i}(t)$ for each actor, indicating whether at time $t$, actor $i$ has moved $\left(A_{i}(t)=1\right)$ or not $A_{i}(t)=0$. Once $A_{i}(t)=1$, the actor is no longer permitted to move. This is to fit the credible constraint that people move at most once in each interval. From standard properties of exponential distributions (see also the definition of rates in SAOMs for social networks, Snijders, 2001), the probability at time $t$ that actor $i \in \mathscr{A}_{t}=\left\{i \in \mathscr{I}: A_{i}(t)=0\right\}$ is the first person to move is

$$
\frac{\lambda_{i}(Y(t), t, \theta)}{\sum_{i \in \mathscr{A}_{t}} \lambda_{i}(Y(t), t, \theta)}
$$

We make the explicit assumption that the rates of moving out are not confounding the discrete choice model for the destination. Thus we tacitly disallow individuals deciding to move because they have decided where to move to.

For computational purposes, we further make the simplifying assumption that $\lambda_{i}(Y(t), t, \theta)=\lambda_{i}(\theta)$ and that these rates may only depend on individual-level covariates and time-constant characteristics of the neighbourhoods. This simplification means that independently for each $i, \operatorname{Pr}\left(A_{i}(t)=1\right)=\operatorname{Pr}\left(T_{i} \leq t\right)=1-e^{-t \lambda_{i}(\theta)}$ and $\operatorname{Pr}\left(A_{i}(t)=0\right)=\operatorname{Pr}\left(T_{i}>t\right)=e^{-t \lambda_{i}(\theta)}$. Assuming that the system is not 'full', i.e. that there is always somewhere to move to, this means that the parameters of the moving out process may be estimated independently of the destination model. In particular, with $\lambda_{i}=e^{\eta_{i}}$, for some linear function $\eta_{i}$ of fixed covariates, this model formulation implies that $\operatorname{cog} \log \left(\operatorname{Pr}\left(A_{i}(t)=1\right)\right)=\eta_{i}$, where cloglog $(\cdot)$ is the complimentary log-log link function. Note that this simplification does not imply that the time-ordering does not matter. Individuals with higher rates are likely to move earlier and the order of moves consequently has to be weighted in the course of estimating the destination process.

Should $\lambda_{i}(\cdot)$ be allowed to depend on time-varying characteristics, then the rates would have to be updated every time anyone moves from a neighbourhood. However, while neighbourhood characteristics can be accounted for by including a 
'lagged' measure, e.g. the ethic composition at the start of a period, this assumption prevents us from investigating phenomena such as 'white flight' (Crowder and South, 2008) in the same detail as the destination processes (e.g. 'white avoidance'). Note that the simplifying assumptions for the decision to move will not systematically bias our analysis of the destination choice as long as the assumption that the processes are not confounded is true.

\subsection{Example statistics}

We will focus here on defining elements of the discrete-choice model for the destinations of moves. We can break the effects down into properties of the actors, origins, potential destinations, as well as combinations of these. As the origin of $i$ at the time $t_{i}$ of a change $j^{*}=j\left(Y_{i \bullet}(s)\right), s<t$, is fixed in evaluating the decisions $\Omega(\mathscr{J} ; Y(t))$, effects in $f_{i}$ cannot depend on $j^{*}$ only. Similarly, effects in $f_{i}$ cannot depend only on properties of $i$. However, all interactions between properties of $j^{*}$ and $i$ on the one hand and properties of $j \in \Omega(\mathscr{J} ; Y(t))$ are admissible. Some effects relevant to our empirical example are the following.

Destination capacity and popularity A natural restriction on movements is availability of housing - no matter how desirable a neighbourhood, if there is no housing no move is possible. We call this the 'capacity' $c_{j}$ of $j$ at time $t$. We may take the capacity into account through the objective function $f_{i}$. The sequential nature of the model means that moves will free up accommodation in the sense that the move will increase the utility for others and thereby create vacancy chains (White, 1970). A potential effect in the opposite direction is that popular neighbourhoods may become more popular (a Matthew effect, see Merton, 1968; and de Solla Price, 1976). We may combine these effects in a statistic in $f_{i}$ that makes sure that the sizes of the neighbourhoods do not fluctuate wildly over time and also prevents heaping in popular neighbourhoods.

It is clear from data over time however that the number of residents remain relatively fixed. This may be reflected by a stricter use of capacity, namely that $\Omega(\mathscr{J} ; Y(t))=\left\{j \in \mathscr{J}: Y_{+j}<c_{j}\right\}$. The utility for $j$ given that $j \in \Omega(\mathscr{J} ; Y(t))$ should reflect the increasing utility of availability as reflecting market prices as well as a decreasing utility of availability as reflecting decreased popularity (the Yogi Berra effect, Hedström, 1998). In addition to the restrictions on $\Omega$, we model the effect of occupancy as a decaying utility of available housing

$$
S_{i j}(t)=\exp \left\{-\alpha \min \left(c_{j}-y_{+j}(t), c\right)\right\}
$$

where $c$ is some truncation point set for computational convenience.

Prices of housing and household assets To reflect differences in the prices of housing, we may include as a statistic $S_{i j}(t)=w_{j}(t)$, where $w_{j}(t)$ is the average log price or another appropriate measure. Instead, or in addition, we may here have a 
compositional variable $S_{i j, \text { comp }}(t)=\sum_{i} y_{k j}(t) v_{k}(t) / y_{+j}$ for some measure of household assets $v_{k}$.

Assuming that housing costs $w_{j}$ and household assets $v_{k}$ are measured on comparable scales, household-area pricing discrepancy can me modelled using the effect

$$
S_{i j, \text { diff }}(t)=\left(v_{I}(t)-w_{j}(t)\right)^{2}
$$

Instead of $w_{j}$ we may use average income $S_{i j \text {,comp }}(t)$, which comprises two aspects, the compositional aspect and a pragmatic proxy for cost of housing. Additionally, we may consider income differences between neighbourhoods $\left(S_{i j^{*}, \text { comp }}(t)-\right.$ $\left.S_{i j \text {, comp }}(t)\right)^{2}$.

Ethnic mixing To model ethnic mixing, let $v_{i}$ indicate if $i$ belongs to a minority ethnic group or not (the extension to multiple categories is straightforward), and we can model mixing through:

$$
S_{i j}(t)=1-\left|v_{i}(t)-\frac{\sum_{k} y_{k j}(t) v_{k}(t)}{y_{+j}(t)}\right|
$$

A positive parameter means that individuals tend to associate higher utility with areas with high proportion of inhabitants of the same ethnic group.

Spatial embedding A plausible factor in moves is the spatial embedding of neighbourhoods. On the one hand, neighbourhoods that are more central may be more attractive, on the other hand there are considerable transaction costs associated with moves over large distances. We focus here only on the latter factor and define a statistic

$$
S_{i j, 5}(t)=\log \left\|w_{j}-w_{j^{*}}\right\|^{2}
$$

where $j^{*} \in \mathscr{J}$ is the current area of $i$. For longitudinal networks it is common to use distances on a log-scale (Preciado et al., 2012; Koskinen et al., 2015), something which may be may be motivated through is relation to the attenuated power-law form of spatial decay (see further e.g. Daraganova et al., 2012).

Kinship and social networks The literature suggests that information is not symmetrically disseminated and that considerable advantages may be had through extramarket structures (Granovetter, 1973). While networks of acquaintances, work colleagues etc, are difficult to gather data on, we may have register data on kinship networks. A simplistic approach for modelling a preference for moving to neighbourhoods where you have relatives is to include the effect $S_{i j, 6}(t)=\sum_{k} y_{k j}(t) a_{i k}$ that is large for areas where $i$ has others $k$ that are kin.

Dynamic endogeneity The incremental nature of the model captures two aspects of endogeneity and emergence. Firstly, the move of $i$ from $j^{*}$ to $j$ implies that any compositional variables for areas are updated by removing the contribution of $i$ to $j^{*}$ and giving $j$ the additional contribution of $i$. A compositional change in the ethnic composition of a neighbourhood thus comes about by successive updates to the 
neighbourhood ethnic composition, for example ethnic minorities moving into a neighbourhood, incrementally increasing the proportion of ethnic minorities.

Second, the conditional nature of the model means that we may include path dependencies. The simplest form of path dependency is the first-order, whereby we model the probability that an individual moves into a neighbourhood $j$ given that the individual currently lives in neighbourhood $j^{*}$.

Similar to homophily and influence effects in stochastic actor-oriented models for social network evolution (Snijders, 2001), there may be strong effects of segregation without there being any evidence in the changes of the marginal distributions of ethnic composition. If there is no effect of segregation, we expect that the ethnic compositions of neighbourhoods will tend to be evenly distributed across networks, everything else equal. If however there is already segregation initially (as reflected in unequal distribution of ethnic proportions), a strong effect for segregation may be needed to maintain the distribution of ethnic composition across neighbourhoods (for longitudinal exponential random graph models this has been addressed through joint modelling of initial conditions and dynamics; Koskinen et al., 2015).

\section{Estimation issues}

Given an observed sequence of moves by individuals with known attributes, the likelihood is tractable and inference is straightforward. In the case that data are only observed, say, once every year, the sequence of moves is not observed. Had utilities been independent of past moves and constant over time, the exact sequence might have been of minor importance. Here, the order of moves is potentially important and while it is unobserved, the composition of areas and individual attributes may bias transitions in an informative way. Of particular interest in the case of moves are the restrictions imposed by capacity of areas (White, 1970).

Given that the process is equivalent to that of Koskinen and Edling (2012) with added constraints, a straightforward modification of the Bayesian inference procedure proposed by Koskinen and Snijders (2007) applies. In particular, given that actors may only move once in any given time-interval, means that the Bayesian data-augmentation scheme can be considerably simplified. To propose updates to the unobserved sequence we only need to shuffle the order of moves. Here, we pick two changes and propose swapping their order provided this does not lead to a violation of the maximal capacity of any neighbourhood.

\section{Empirical illustration}

The Figure 1 illustrates the structure of the movements on the Stockholm housing market. The 128 nodes represent the neighbourhoods defined by the small area market statistics of Statistics Sweden. 
Fig. 1 Movement flows among 128 Stockholm neighbourhoods (based on small area market statistics) aggregated across 1990-2003 (directionality has been suppressed for visibility). In the period 1990-1991 there were 572,389 households across the 128 areas and 41,578 moves. The thickness and saturation of a line is proportional to the number of moves between respective areas (code from spatial.ly gratefully acknowledged)

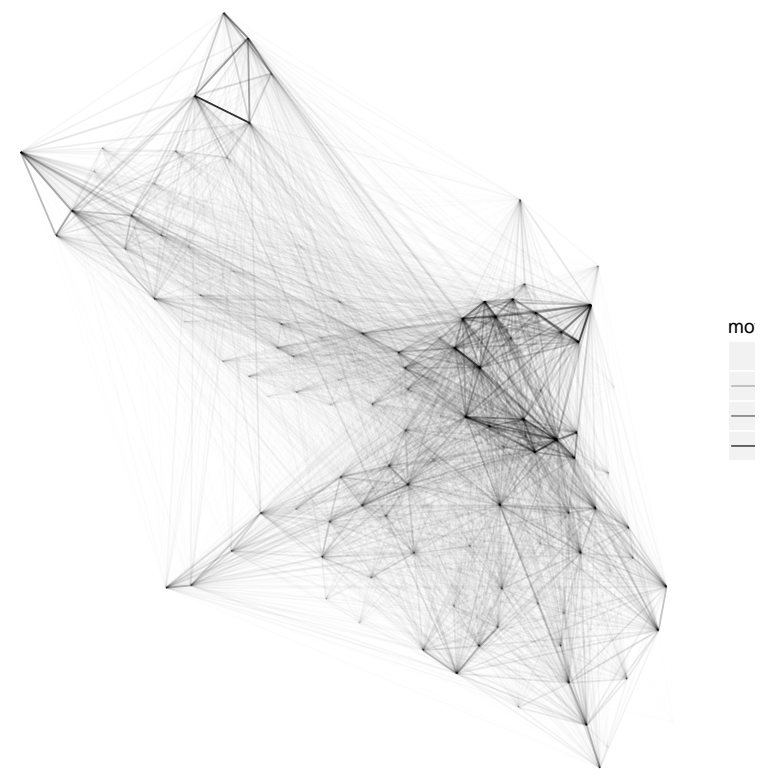

We consider here a simplified model where we only use transitions in the period 1990-1991. In total there were 572,389 actors (households) in the Stockholm area in this period and a total of 41,578 actors were registered at different addresses in 1990 and 1991. The main focus of interest is to explain ethnic segregation on the housing market as measured by the different proportions of actors that were either second or first generation immigrants in neighbourhoods. The overall proportion of thus defined immigrants in the Stockholm area was $22.4 \%$. We model segregation by including the two effects 'mover-dest immig sim' and 'orig-dest im diff '. The former is defined as Eq. 2 and the latter as $\left|\sum_{i} y_{i j} v_{i}(t) / y_{i j}-\sum_{i} y_{+j} v_{i}(t) / y_{+j}\right|$, which measures the dissimilarity in proportion of immigrants in the origin and potential destination neighbourhood of $i$. To control for income distribution, two effects 'origdest inc diff', $\left(S_{i j^{*}, \text { comp }}(t)-S_{i j, \text { comp }}(t)\right)^{2}$, and 'mover-dest income diff', $\left(v_{j}(t)-\right.$ $\left.S_{i j, \text { comp }}(t)\right)^{2}$, were constructed.

To capture the potential of vacancy chains the capacity $c_{j}$ of each neighbourhood is set to $100.05 \%$ of the total number of inhabitants in 1991. Thus a neighbourhood that increases its population by $0.05 \%$ early on in the period is effectively removed from the choice-set of actors until someone moves out of this neighbourhood. The scaling parameter in Eq. 1 is set to $\alpha=0.25$ which corresponds to a slightly less rapid decade than a factor $1 / 2$. Given that the largest difference in capacity and number of inhabitants is about 8 , we set $c=19$ as an arbitrary cut-off after which the function is essentially flat. The gradient could in principle be estimated by treating $\alpha$ as a free parameter but we have not pursued this here. This is partly because the capacities have not been informed by physical availability of housing. 
For this example we have not estimated the moving-out process nor have we taken into account actors entering or leaving the Stockholm market. Estimation results from a Markov chain Monte Carlo algorithm based on a modified version of Koskinen and Snijders (2007) using 5,000 iterations are provided in Table 1.

Table 1 Posterior summaries for SAOM fitted to the movement flows on the Stockholm Housing market between 1990-2003

\begin{tabular}{lll}
\hline Effect & Parameter mean & Std \\
\hline capacity & -2.945 & 0.027 \\
dest income & -5.409 & 0.508 \\
orig-dest inc diff & -2.396 & 5.089 \\
mover-dest income diff & -10.890 & 0.744 \\
orig-dest im diff & -3.500 & 0.145 \\
mover-dest immig sim & 1.292 & 0.048 \\
orig-dest distance & -128.725 & 1.423 \\
\hline
\end{tabular}

The negative capacity effect could be interpreted as popular neighbourhoods becoming more popular, or equivalently, the further they are from capacity, the less attractive they are. However, the proxy for capacity is blunt and its interpretation in terms of popularity could be argued to be somewhat circular.

There is no discernible effect of neighbourhood homophily on income level but a clear preference for actors moving to neighbourhoods with a similar average income level to their own. The negative coefficient for distance indicates that actors prefer to move only short distances. Over and above these other effects, there are two clear segregation effects: actors move between neighbourhoods with similar proportions of immigrants (coef: -3.5 ); and immigrant actors prefer to move to neighbourhoods that have high proportion of immigrants (coef: 1.29) (and conversely, non-immigrants prefer low proportion of immigrants).

The relative effect of vacancy chains is indicated by the proportion of proposed permutations in the course of estimation that would have resulted in a neighbourhood exceeding its capacity. Here, in $30 \%$ of proposals for draws from the full conditional posterior of the unobserved sequence of moves, given the rest, an actor was attempting to move to a neighbourhood that had no vacancy.

\section{Conclusions and future directions}

We presented a generative model for modelling allocation of individuals to neighbourhoods while taking systemic dependencies into account. For the example of the Stockholm housing market we found that there is clear evidence of households sorting themselves according to ethnic background, sustaining and reproducing ethnic segregation on the housing market. As one of the key purposes is to generate predictions under various scenarios we need to specify the moving-out process in 
order to fully leverage the generative capacity of the model. Furthermore, applying the model to data across 13 consecutive years, gives us an opportunity to investigate how the model changes over time. This could be done by drawing on work on change-point analysis for sequential models (McCormick, et al., 2012) or by specifying a hierarchical model as in Koskinen, Caimo, and Lomi (2015) and model a smooth change to the parameters as a function of time. Further modifications might include accounting for moves into and out of $\mathscr{I}$ as well as empirically determined capacities.

There are obvious connections to demographic micro-simulation techniques ( $\mathrm{Li}$ and O'Donoghue, 2013) but, as pointed out by Snijders and Steglich (2013), the inferential framework of the actor-oriented model allows a simulation model that is informed by real data. As the modelling of housing moves demonstrates, the principles of Snijders' (2001) stochastic actor-oriented model provides a general framework that applies much more widely than to just social networks.

Acknowledgements The research leading to these results has received funding from the European Research Council under the European Union's Seventh Framework Programme (FP7/20072013) / ERC grant agreement no 324233, Riksbankens Jubileumsfond (DNR M12-0301:1), and the Swedish Research Council (DNR 445-2013-7681) and (DNR 340-2013-5460)

\section{References}

1. Abdulkadiroğlu, Atila and Sönmez, Tayfun: School choice: a mechanism design approach. American Economic Review, 93, 729-747 (2003)

2. Aldous, D.: Minimization algorithms and random walk on the d-cube. Ann. Probab., 11, 403413 (1983)

3. Butts, C.T.: Models for generalised location systems . Sociological Methodology, 37, 283348 (2007)

4. Crowder, K. and South, S. J.: Spatial dynamics of white flight: the effects of local and extralocal racial conditions on neighborhood out-migration, American Sociological Review, 73, 792-812 (2008)

5. Daraganova, G., Pattison, P., Koskinen, J., Mitchell, B., Bill, A., Watts, M., Baum, S.: Networks and geography: modelling community network structures as the outcome of both spatial and network processes. Social Networks, 34, 6-17 (2012)

6. Granovetter, M.S.: The strength of weak ties? Am. J. Sociol., 78, 1360-1380 (1973)

7. Hedström, P.: Rational imitation. In: Hedström, P, and Swedberg, R. (eds.) Social mechanisms: An analytical approach to social theory, pp. 306-327. Cambridge University Press, MA (1998)

8. Li, Jinjing, and O'Donoghue, C.: A survey of dynamic microsimulation models: uses, model structure and methodology. International Journal of Microsimulation, 6, 3-55 (2013)

9. Koskinen J., Caimo, A., Lomi, A.: Simultaneous modeling of initial conditions and time heterogeneity in dynamic networks: An application to Foreign Direct Investments. Network Science, 3, 58-77 (2015)

10. Koskinen, J. and Edling, C.: Modelling the evolution of a bipartite network - peer referral in interlocking directorates. Social Networks, 34, 309-322 (2012)

11. Koskinen, J.H., Snijders, T.A.B.: Bayesian inference for dynamic social network data. J. Stat. Plan. Inference, 137, 3930-3938 (2007) 
12. Maddala, G.S.: Limited-dependent and qualitative variables in Econometrics. Cambridge University Press, Cambridge (1983)

13. McCormick, T.H., Raftery, A.E., Madigan, D., Burd, R.S.: Dynamic logistic regression and dynamic model averaging for binary classification. Biometrics, 68, 23-30 (2012)

14. Merton, R.K.: The Matthew effect in science. Science, 159, 56-63 (1968)

15. Preciado, P., Snijders, T.A.B., Burk, W.J., Stattin, H., Kerr, M.: Does proximity matter? Distance dependence of adolescent friendships. Soc. Netw., 34, 18-31 (2012)

16. Snijders, T.A.B.: Statistical methods for network dynamics. In: Luchini, S.R. (ed.) XLIII Scientific Meeting, Italian Statistical Society, pp. 281-296. CLEUP, Padova (2006)

17. Snijders, T.A.B.: The statistical evaluation of social network dynamics. Sociological Methodology, 31, 361-395 (2001)

18. Snijders, Tom AB, and Christian EG Steglich: Representing micro-macro linkages by actorbased dynamic network models. Sociological methods \& research, 44, 222-271 (2015)

19. de Solla Price, D.: A general theory of bibliometric and other advantage processes. American Society for Information Science, 27, 292-306 (1976)

20. White, H.: Chains of Opportunity. System Models of Mobility in Organizations. Harvard University Press, Cambridge, MA (1970) 